\title{
BOUNDARY TANGENTIAL CONVERGENCE ON SPACES OF HOMOGENEOUS TYPE
}

\author{
PATRICIO CIFUENTES, JOSÉ R. DORRONSORO AND JUAN SUEIRO
}

\begin{abstract}
We study tangential convergence of convolutions with approximate identities of functions defined on a homogeneous type space and having a certain regularity. Our results contain those already known for the Euclidean case and give new ones for stratified nilpotent Lie groups and for solutions of the Dirichlet problem on Lipschitz domains.
\end{abstract}

\section{INTRODUCTION}

Given an $L^{p}$ function $f$, it is very well known that its Poisson integral $u(y, t)=P_{t} * f(y)$ converges nontangentially (i.e. inside cones $\Gamma(x)=\{(y, t)$ : $|y-x|<(1+\lambda) t\})$ to $f(x)$ for a.a. $x \in \mathbb{R}^{N}$; it is also very well known that convergence fails when the approach regions have a certain degree of tangentiality.

However, when $f$ has certain regularity, tangential convergence does indeed hold. More concretely, defining

$$
\begin{gathered}
\Omega_{\gamma, \lambda}(x)=\left\{(y, t):|y-x|<(1+\lambda) t^{\gamma}\right\}, \\
T_{\gamma, \lambda} f(x)=\sup \left\{|u(y, t)|=\left|P_{t} * f(y)\right|:(y, t) \in \Omega_{\gamma, \lambda}(x)\right\},
\end{gathered}
$$

Nagel, Rudin and Shapiro prove that for $f$ in the space $W^{p, a}=\left\{J_{a} * F: F \in\right.$ $\left.L^{p}\right\}$ of Bessel potentials of $L^{p}$ functions, $1<p<\infty$, ap $<N$, the inequality

$$
\left\|T_{1-a p / N, \lambda} f\right\|_{p} \leq C\|f\|_{W^{p, a}},
$$

holds for a constant $C$ independent of $f$. (See [NRS]; there is also an analogous result when $a p=N$.) Their argument relies on a weak type inequality combined with the so called strong capacitary estimates.

Later on, A. Nagel and E. M. Stein [NS] introduced a new approach to the study of tangential convergence. Given a function $F(y, t), y \in \mathbb{R}^{N}, t>0$, they defined weighted tangential maximal operators

$$
M_{a, \gamma, p}^{\delta} F(x)=\sup \left\{\delta^{N / p} t^{a}|F(y, \delta t)|:(y, t) \in \Omega_{\gamma, \lambda}(x), \delta t<t^{\gamma}\right\},
$$

for which they proved

$$
\left\|M_{a, 1-a p / N, p}^{\delta} F\right\|_{p} \leq C\|N F\|_{p}
$$

Received by the editors April 30, 1990.

1980 Mathematics Subject Classification (1985 Revision). Primary 31B25; Secondary 46E35.

The first author was partially supported by the DGICyT, grant PS87-0027, the second author by the CICyT, grant PB87-0127, and the third by the DGICyT, grant PS87-0027. 
where $C$ is independent of $\delta$. Since they also show, using special identities for Bessel functions, that for $f=J_{a} F$ and $U=P_{t} * F$,

$$
T_{\gamma, \lambda} f(x) \leq C\left(M f(x)+\sum_{0}^{\infty} 2^{k(a-N / p)} M_{a, \gamma, p}^{2^{k}} U(x)\right),
$$

if $a p<N$ one recovers the $L^{p}$ estimate for $T_{1-a p / N, \lambda} f$.

Until now we have only considered regions with the maximum degree of tangentiality, i.e., $\gamma=1-a p / N$. When $1-a p / N<\gamma<1$, P. Ahern and A. Nagel proved [AN] that if $m \gamma=N-a p$ and $\mu$ is a measure on a space of homogeneous type such that $\mu(B(x, r)) \leq C r^{m}$, then

$$
\left\|M_{a, \gamma, p}^{1} F\right\|_{L^{p}(d \mu)} \leq C\|N F\|_{p}^{p}
$$

(the general case in $\mathbb{R}^{N}$ follows by dilation), and as a consequence they obtain tangential convergence results with respect to the $\Omega_{\gamma, \lambda}$ with the exceptional sets having now zero $(N-a p) / \gamma$-Hausdorff measure for functions in $W^{p, a}$ or in the Besov spaces $B_{a}^{p, p}$.

Tangential convergence has also been studied in the unit ball of $\mathbb{C}^{N}[\mathrm{Go}, \mathrm{Su}]$ and for other spaces of regular functions [Do]. In this paper we will consider the situation in the setting of the spaces of homogeneous type, proving abstract tangential convergence results for certain spaces of "regular" functions which in the $\mathbb{R}^{N}$ case contain the ones mentioned above, proceeding then to give applications to functions on stratified nilpotent Lie groups and to solutions of Dirichlet problems on Lipschitz domains.

More specifically, in $\S 2$, using the method in [Su], we prove a strong $L^{p}$ estimate for weighted tangential maximal functions on spaces of homogeneous type with respect to Hausdorff content, rather than singular measures, as was the case in [AN and Su]. We thus avoid the need of a general Frostman lemma.

In $\S 3$ we define the classes $C^{p, a}$ of "regular" functions on spaces of homogeneous type by means of an abstract notion of local approximation by averaging operators, and we prove strong $L^{p}$ estimates with respect to Hausdorff content for tangential maximal functions of "Poisson integrals," i.e., convolutions with approximate identities of dilation type, of these functions.

We consider in $\S 4$ stratified nilpotent Lie groups and prove that the Sobolev spaces associated to their Lie algebra are continuously imbedded in the spaces $C^{p, a}$ defined by means of suitable averaging operators. As a consequence, we obtain in particular tangential convergence results for harmonic functions on generalized half-spaces and on symmetric spaces, and for Cauchy-Szegö and Poisson-Szegö integrals on the Siegel domains lying "above" Heisenberg groups.

Section 5 is concerned with Dirichlet problems on Lipschitz domains with boundary data in the Sobolev space $W^{p, 1}$ for whose solutions we prove tangential convergence except on an exceptional set of appropriate Hausdorff dimension. The results of $\S 3$ cannot be applied here directly, since in general one lacks the necessary estimates for the Poisson kernel, and we make instead use of the deep estimates for harmonic measure and the kernel function due to Caffarelli, Dahlberg, Fabes, Jerison and Kenig among others (see [CFMS, Da 1 and $\mathrm{JK}]$ ). Although we will restrict our exposition to the Laplacian, the same results also apply to more general second order elliptic operators in divergence form. 
Finally, in $\S 6$ we discuss the situation when ap equals the homogeneous dimension (when $a p$ is strictly greater than the dimension, the spaces $C^{p, a}$ contain only, as it happens in the $\mathbb{R}^{N}$ case, continuous functions), where we will prove a weak type estimate for the tangential maximal operators. The section ends with a brief discussion on nontangential convergence.

\section{TANGENTIAL MAXIMAL FUNCTIONS}

In this section we study certain tangential maximal operators $M_{a, \gamma, p}^{\delta}$ on spaces of homogeneous type. We prove a strong $L^{p}$ estimate with respect to Hausdorff content (Theorem 2.1). This result is related to Theorem $4^{\prime}$ in [NS], Theorem 6.1 and Lemma 6.2 in [AN], and Theorem 1.7 and Propositions 1.12 and $3.5 \mathrm{in}[\mathrm{Su}]$. We shall follow the method of $[\mathrm{Su}]$ in order to prove Theorem 2.1. It may be interesting to notice that the argument of [NS and AN], based on an atomic decomposition for tent spaces, does not seem to work here, the difficulty being that we are dealing with Hausdorff content rather than with a true measure.

We shall work in a space of homogeneous type $(\mathbf{X}, \mathrm{d}, \mu)$ (see [CW] for more information on these spaces). Thus, $\mathbf{d}$ is a quasidistance on $\mathbf{X}$, i.e., it satisfies, in addition to the other usual properties of a distance, a quasitriangle inequality $\mathrm{d}(x, z) \leq K(\mathrm{~d}(x, y)+\mathrm{d}(y, z))$ with constant $K \geq 1$. We shall assume that the Borel measure $\mu$ is $Q$-homogeneous for some $Q>0$. This means that, writing $|E|$ for the $\mu$-measure of the set $E$, we have

$$
|B(x, r)|=|\{y \in \mathbf{X}: \mathrm{d}(y, x)<r\}| \simeq r^{Q},
$$

that is $C r^{Q} \leq|B(x, r)| \leq r^{Q} / C$ for some constant $C>0$ (here and in what follows, $C$ will stand for any absolute numerical constant whose concrete value may vary on each appearance).

We recall now the definition of Hausdorf content $H_{\infty}^{m}$, and Hausdorff measure $H^{m}$ of dimension $m, 0<m \leq Q$. For $0<\rho \leq \infty$, and $E$ a subset of $\mathbf{X}$, write

$$
H_{\rho}^{m}(E)=\inf \left\{\sum_{i} r_{i}^{m}: E \subset \bigcup_{i} B\left(x_{i}, r_{i}\right), r_{i} \leq \rho\right\},
$$

and

$$
H^{m}(E)=\sup _{\rho>0} H_{\rho}^{m}(E)=\lim _{\rho \rightarrow 0} H_{\rho}^{m}(E) .
$$

The following facts are easy consequences of the definitions:

$$
\begin{gathered}
H_{\infty}^{m}\left(\bigcup_{i=1}^{\infty} E_{i}\right) \leq \sum_{i=1}^{\infty} H_{\infty}^{m}\left(E_{i}\right) ; \\
H_{\infty}^{m}(B(x, r)) \simeq r^{m} ; \\
H^{m}(E)=0 \quad \text { if and only if } H_{\infty}^{m}(E)=0 .
\end{gathered}
$$

In the upper half-space $\mathbf{X} \times(0, \infty)$ we define the following "approach regions" to the "boundary" $\mathbf{X}$. For $0<\gamma \leq 1, \lambda \geq 0$ and $x \in \mathbf{X}$ set

$$
\Omega_{\gamma, \lambda}(x)=\left\{(y, r) \in \mathbf{X} \times(0, \infty): \mathrm{d}(x, y) \leq(1+\lambda) r^{\gamma}, r \leq 1\right\} .
$$

Notice that for $\mathbf{X}=\mathbb{R}^{N}$, the region $\Omega_{1, \lambda}(x)$ is the usual nontangential cone $\Gamma_{\lambda}(x)$, whereas if $0<\gamma<1, \Omega_{\gamma, \lambda}(x)$ is a tangential approach region at $x$, 
making contact of order $1 / \gamma$. For general $\mathbf{X}$ we still use these two adjectives although their geometrical meaning is no longer present. In what follows we will assume $\lambda$ to be fixed once and for all. Associated to these regions we define the nontangential and (weighted) tangential maximal functions of an $F: \mathbf{X} \times(0, \infty) \rightarrow \mathbb{C}$ as follows: for $\delta \geq 1, a \geq 0,0<p<\infty$, and $x \in \mathbf{X}$, set

$$
N F(x)=\sup _{(y, r) \in \Gamma_{\lambda}(x)}|F(y, r)|
$$

and

$$
M_{a, \gamma, p}^{\delta} F(x)=\sup _{\substack{(y, r) \in \Omega_{\gamma, \lambda}(x) \\ \delta r \leq r^{\gamma}}} \delta^{Q / p} r^{a}|F(y, \delta r)| .
$$

We now have

Theorem 2.1. Assume $0<p<\infty, \delta \geq 1, a \geq 0,0<\gamma<1,0<m \leq Q$, and $m=(Q-a p) / \gamma$. Then

$$
\left\|M_{a, \gamma, p}^{\delta} F\right\|_{L^{p}\left(H_{\infty}^{m}\right)} \stackrel{\text { def }}{=}\left(\int_{0}^{\infty} H_{\infty}^{m}\left(\left\{M_{a, \gamma, p}^{\delta} F>t\right\}\right) t^{p-1} d t\right)^{1 / p} \leq C\|N F\|_{p}
$$

where the constant $C$ is independent of $\delta$ and \|\|$_{p}$ stands for the $L^{p}$ norm with respect to $\mu$.

Proof. Without loss of generality, assume $F$ is nonnegative. Notice that

$$
M_{a, \gamma, p}^{\delta} F(x)=\left(M_{a p, \gamma, 1}^{\delta}\left(F^{p}\right)(x)\right)^{1 / p},
$$

so it suffices to prove the theorem in the case $p=1$. Setting $\delta^{*}=\delta^{(m-Q) \gamma /(1-\gamma)}$, it is readily seen that $\delta r \leq r^{\gamma}$ implies $\delta^{Q} r^{a} \leq \delta^{*}$, since $m=(Q-a) / \gamma$. For $\tau>0$ and $\alpha>0$ define

$$
E(\tau, \alpha)=\left\{x \in \mathbf{X}: \exists(y, r) \in \Omega_{\gamma, \lambda}(x), \delta r \leq r^{\gamma}, F(y, \delta r)>\tau, \delta^{Q_{r}}>\alpha\right\} ;
$$

then, $E(\tau, \alpha)$ is empty if $\alpha>\delta^{*}$.

The theorem will be an immediate consequence of the following two lemmas which relate the size of the sets $E(\tau, \alpha)$ to the distribution functions of $M_{a, \gamma, 1}^{\delta} F$ and $N F$.

Lemma 2.2. There exists an absolute constant $D$ such that

$$
\int_{0}^{\infty} H_{\infty}^{m}\left(\left\{M_{a, \gamma, 1}^{\delta} F>t\right\}\right) d t \leq D \int_{0}^{\infty} d \tau \int_{0}^{\delta^{*}} H_{\infty}^{m}(E(\tau, \alpha)) d \alpha .
$$

Proof. If $M_{a, \gamma, 1}^{\delta} F(x)>t>0$, then $\delta^{Q_{r}} F(y, \delta r)>t$ for some $(y, r) \in$ $\Omega_{\gamma, \lambda}(x)$ with $\delta r \leq r^{\gamma}$. Let $j$ and $k$ be integers so that

$$
2^{j} \delta^{*}<\delta^{Q_{r}} \leq 2^{j+1} \delta^{*}, \quad 2^{k}<F(y, \delta r) \leq 2^{k+1} ;
$$

in particular, $j \leq-1$. Then $x \in E\left(2^{k}, 2^{j} \delta^{*}\right), 2^{k+1} 2^{j+1} \delta^{*}>t$ and thus, by $(1)$,

$$
H_{\infty}^{m}\left(\left\{M_{a, \gamma, 1}^{\delta} F>t\right\}\right) \leq \sum_{2^{k+j} \delta^{*}>t / 4} H_{\infty}^{m}\left(E\left(2^{k}, 2^{j} \delta^{*}\right)\right)
$$


Therefore,

$$
\begin{aligned}
\int_{0}^{\infty} H_{\infty}^{m}\left(\left\{M_{a, \gamma, 1}^{\delta} F>t\right\}\right) d t & \leq \sum_{k=-\infty}^{\infty} \sum_{j=-\infty}^{-1} \int_{0}^{2^{k+j+2} \delta^{*}} H_{\infty}^{m}\left(E\left(2^{k}, 2^{j} \delta^{*}\right)\right) d t \\
& =4 \sum_{k=-\infty}^{\infty} 2^{k} \sum_{j=-\infty}^{-1} 2^{j} \delta^{*} H_{\infty}^{m}\left(E\left(2^{k}, 2^{j} \delta^{*}\right)\right) \\
& \leq D \int_{0}^{\infty} d \tau \int_{0}^{\delta^{*}} H_{\infty}^{m}(E(\tau, \alpha)) d \alpha
\end{aligned}
$$

Lemma 2.3. There is a constant $C$ depending only on the space $\mathbf{X}$ such that

$$
\int_{0}^{\delta^{*}} H_{\infty}^{m}(E(\tau, \alpha)) d \alpha \leq C|\{N F>\tau\}| .
$$

Proof. Let $\mathscr{B}=\left\{B_{k}\right\}=\left\{B\left(x_{k}, r_{k}\right)\right\}$ be a Whitney decomposition (see [CW]) of $N(\tau)=\{N F>\tau\}$, that is, a family of balls in $\mathbf{X}$ verifying (i) $N(\tau)=\bigcup_{k} B_{k}$; (ii) the balls $B_{k}$ are $h$-disjoint, i.e., no point of $\mathbf{X}$ lies in more than $h$ distinct $B_{k}$; (iii) $B\left(x_{k}, h r_{k}\right)$ is not contained in $N(\tau)$. The constant $h$ depends only on the space $\mathbf{X}$.

If $x \in E(\tau, \alpha)$ there is some $(y, r) \in \Omega_{\gamma, \lambda}(x)$ with $\delta r \leq r^{\gamma}$ so that $F(y, \delta r)>\tau$ and $\delta^{Q_{r}}{ }^{a}>\alpha$. Then, $B(y, \delta r) \subset N(\tau)$, so $y \in B_{k}$ for some $k$. By (iii), there exists $z \in B\left(x_{k}, h r_{k}\right)-N(\tau)$ (set difference); so, $z \notin B(y, \delta r$ ) and thus, by the triangle inequality,

$$
\delta r<\mathrm{d}(z, y) \leq K\left(\mathrm{~d}\left(z, x_{k}\right)+\mathrm{d}\left(x_{k}, y\right)\right) \leq K(h+1) r_{k}=h^{\prime} r_{k} .
$$

Therefore

$$
\begin{aligned}
\mathrm{d}\left(x, x_{k}\right) & \leq K\left(\mathrm{~d}(x, y)+\mathrm{d}\left(y, x_{k}\right)\right) \leq K\left(r^{\gamma}+r_{k}\right) \\
& \leq K\left(\left(\frac{h^{\prime} r_{k}}{\delta}\right)^{\gamma}+r_{k}\right) \leq C_{1}\left(\left(\frac{r_{k}}{\delta}\right)^{\gamma}+r_{k}\right),
\end{aligned}
$$

with $C_{1}=K h^{\prime} \geq K\left(h^{\prime}\right)^{\gamma}$.

Write $\mathscr{B}$ as $\mathscr{B}^{1} \cup \mathscr{B}^{2}$, with $\mathscr{B}^{1}=\left\{B_{k}: r_{k} \leq\left(r_{k} / \delta\right)^{\gamma}\right\}$ and $\mathscr{B}^{2}=\left\{B_{k}: r_{k}>\right.$ $\left.\left(r_{k} / \delta\right)^{\gamma}\right\}$. Since $\alpha<\delta^{Q_{r}}{ }^{a} \leq \delta^{Q}\left(h^{\prime} r_{k} / \delta\right)^{a}$, it follows that, if we define $B_{k}^{\prime}=$ $B\left(x_{k}, 2 C_{1}\left(r_{k} / \delta\right)^{\gamma}\right)$ for $B_{k} \in \mathscr{B}^{1}$ and $B_{k}^{\prime}=B\left(x_{k}, 2 C_{1} r_{k}\right)$ for $B_{k} \in \mathscr{B}^{2}$, we have

$$
E(\tau, \alpha) \subset\left(\bigcup_{\substack{B_{k} \in \mathscr{B}^{1} \\ \alpha<C_{2} \delta^{Q-a} r_{k}^{a}}} B_{k}^{\prime}\right) \cup\left(\bigcup_{B_{k} \in \mathscr{B}^{2}} B_{k}^{\prime}\right),
$$

with $C_{2}=h^{\prime Q} \geq h^{\prime a}$ and hence,

$$
\begin{aligned}
\int_{0}^{\delta^{*}} H_{\infty}^{m}(E(\tau, \alpha)) d \alpha & \leq \int_{0}^{\delta^{*}}\left\{\sum_{\substack{B_{k} \in \mathscr{B}^{1} \\
\alpha<C_{2} \delta^{Q-a} r_{k}^{a}}} H_{\infty}^{m}\left(B_{k}^{\prime}\right)\right\} d \alpha+\delta^{*} \sum_{B_{k} \in \mathscr{B}^{2}} H_{\infty}^{m}\left(B_{k}^{\prime}\right) \\
& \leq C \sum_{B_{k} \in \mathscr{B} 1} \delta^{Q-a} r_{k}^{a}\left(r_{k} / \delta\right)^{\gamma m}+C \sum_{B_{k} \in \mathscr{B}^{2}} \delta^{(m-Q) \gamma /(1-\gamma)} r_{k}^{m},
\end{aligned}
$$


by (2) and the definition of $\delta^{*}$. In the first sum, $\delta^{Q-(a+\gamma m)} r_{k}^{a+\gamma m}=r_{k}^{Q}$ for $a+\gamma m=Q$. In the second sum, $\delta^{-\gamma} \leq r_{k}^{1-\gamma}$, so $\delta^{-\gamma /(1-\gamma)} \leq r_{k}$ and

$$
\delta^{-(Q-m) \gamma /(1-\gamma)} r_{k}^{m} \leq r_{k}^{Q-m} r_{k}^{m}
$$

Thus

$$
\int_{0}^{\delta^{*}} H_{\infty}^{m}(E(\tau, \alpha)) d \alpha \leq C \sum_{k} r_{k}^{Q} \leq C \sum_{k}\left|B_{k}\right| \leq C h|\{N F>\tau\}|
$$

\section{TANGENTIAL BEHAVIOR OF REGULAR FUNCTIONS}

We will define regular functions on the space of homogeneous type $\mathbf{X}$ in terms of local approximation by certain averaging functions. More precisely, we assume that for each ball $B \subset \mathbf{X}$ there is a linear operator $A_{B}: L_{\text {loc }}^{1}(\mathbf{X}) \rightarrow C(\mathbf{X})$ satisfying the following properties:

1. Mean dominance. There is a constant $C$ such that for any $f \in L_{\text {loc }}^{1}(\mathbf{X})$, for any ball $B$ and all $y \in B$,

$$
\left|A_{B} f(y)\right| \leq C f_{B}|f| d x,
$$

where the right-hand side stands for $\int_{B}|f| d x /|B|$ and we write $d x$ instead of $d \mu(x)$;

2. Coherence. If $B^{\prime} \subset B$, then $A_{B^{\prime}}\left(A_{B} f\right)=A_{B} f$; in addition, we will assume that for any constant $\lambda$ and any ball $B, A_{B}(\lambda)=\lambda$. Any such family will be called a family of averaging operators and denoted by either $A$ or $\left\{A_{B}\right\}$.

An obvious example is given by the means $A_{B}(f)=f_{B} f d x$; when $\mathbf{X}=\mathbb{R}^{Q}$ and $k \in \mathbb{N}$, a second example is given by the family $\left\{A_{B}^{k}\right\}$ of the orthogonal projections of $L_{\text {loc }}^{1}\left(\mathbb{R}^{Q}\right)$ into the space $\mathbb{P}_{k}(B)$ of restrictions to the ball $B$ of polynomials of degree $\leq k$ endowed with the inner product $(\phi, \psi)=$ $f_{B} \phi(x) \psi(x) d x$ [DVS, p. 8].

Let $A$ be a given family of averaging operators on $\mathbf{X}$, and for $a>0, x \in \mathbf{X}$ and $f \in L_{\text {loc }}^{1}(\mathbf{X})$ define

$$
f_{a, A}^{\#}(x)=f_{a}^{\#}(x)=\sup _{x \in B} \mathrm{r}(B)^{-a} f_{B}\left|f-A_{B} f\right| d y,
$$

where $\mathrm{r}(B)$ denotes the radius of $B$; if $1 \leq p \leq \infty$, we will denote by $C_{A}^{p, a}(\mathbf{X})=C^{p, a}$ the space of those $L^{p}$ functions $f$ such that $f_{a, A}^{\#} \in L^{p}$, endowed with the norm $\|f\|_{p, a}=\|f\|_{p}+\left\|f_{a}^{\#}\right\|_{p}$.

Observe that if $1<p<\infty$, and $A_{B} f=f_{B} f d x, C^{p, 0}=L^{p}$, whereas $C^{\infty, 0}=B M O$. Also, if $0<a \leq 1$ and again $A_{B} f=f_{B} f d x$,

$$
C_{A}^{\infty, a}=\operatorname{Lip}_{a}=\left\{f \in L^{\infty}:|f(x)-f(y)| \leq C_{f} \mathrm{~d}(x, y)^{a}\right\} .
$$

Finally, if $\mathbf{X}=\mathbb{R}^{Q}, 1<p<\infty, a>0, k=[a]$ and $A=\left\{A_{B}^{k}\right\}$, the Sobolev spaces $W^{p, a}$ and the Besov spaces $B_{a}^{p, q}, 1 \leq q \leq p$, are continuously imbedded in $C_{A}^{p, q}\left(\mathbb{R}^{Q}\right)$ [DVS, Chapter 7].

In what follows we will assume that the family $A$ is fixed. 
Proposition 3.1. If $f \in C^{p, a}, 1<r \leq p<Q / a$ and $r^{*}=Q r /(Q-a r)$, for any ball $B$,

$$
\left(\int_{B}\left|f-A_{B} f\right|^{r^{*}} d x\right)^{1 / r^{*}} \leq C\left(\int_{2 B}\left|f_{a}^{\#}\right|^{r} d x\right)^{1 / r} .
$$

If $p=Q / a$ and $p^{\prime}=p /(p-1)$, for some $\beta, C$ and all $f \in C^{p, a}$,

$$
\int_{B} e^{\beta\left(\left|f-A_{B} f\right| /\left\|f_{a}^{*} \chi_{B}\right\|_{p}\right)^{p^{\prime}}} d x \leq C|B| .
$$

Finally, if $p>Q / a, f$ is continuous.

This is an easy consequence of the estimate

$$
\left|f(y)-A_{B} f(y)\right| \leq C^{\prime} \int_{\mathrm{d}(y, z) \leq C r} \mathrm{~d}(y, z)^{a-Q} f_{a}^{\#}(z) d z,
$$

which can be proved as in [Do, p. 676].

Now let $\phi:[0, \infty) \rightarrow[0, \infty)$ be a nonincreasing function such that

$$
\sum_{0}^{\infty} 2^{k Q} \phi\left(2^{k}\right)<\infty
$$

For $x, y \in X, t>0$, and $f \in L^{p}$, set $\Phi_{t}(x, y)=t^{-Q} \phi(\mathrm{d}(x, y) / t)$,

$$
u(x, t)=\int_{X} \Phi_{t}(x, y) f(y) d y
$$

and

$$
T_{\gamma, \lambda} f(x)=\sup \left\{|u(y, t)|:(y, t) \in \Omega_{\gamma, \lambda}(x)\right\} .
$$

The main result of this section is

Theorem 3.2. If $1<p<Q / a, Q-a p<m \leq Q$ and $\gamma=(Q-a p) / m$, then $\left\|T_{\gamma, \lambda} f\right\|_{L^{p}\left(H_{\infty}^{m}\right)} \leq C\|f\|_{p, a}$, for all $f \in C^{p, a}$ and all $\lambda>0$.

We observe that for $\mathbf{X}=\mathbb{R}^{Q}$, we recover the results of [NRS, NS and AN] for Poisson integrals of Sobolev and Besov functions.

This theorem will be an easy consequence of the following two lemmas. Recall that the tangential maximal operator $M_{a, \gamma, p}^{\delta}$ was defined in $\S 2$; we shall write $M_{a, \gamma, p}^{k}$ for $M_{a, \gamma, p}^{2^{k}}$. Mf denotes the Hardy-Littlewood maximal function of $f$.

Lemma 3.3. Set $F(y, t)=f_{B(y, t)} f_{a}^{\#}(z) d z$; then

$$
T_{\gamma, \lambda} f(x) \leq C\left(M f(x)+\sum_{0}^{\infty} 2^{k(a-Q / p)} M_{a, \gamma, p}^{k} F(x)\right) .
$$

Lemma 3.4. If $f \in C^{p, a}$ and $Q-a p<m \leq Q$, then $\|M f\|_{L^{p}\left(H_{\infty}^{m}\right)} \leq C\|f\|_{p, a}$.

Taking these two lemmas for granted, we finish now the proof of Theorem 3.2. Setting $A=\sum_{0}^{\infty} 2^{k(a-Q / p) / 2}$, we have $\left\{\sum_{0}^{\infty} 2^{k(a-Q / p)} M_{a, \gamma, p}^{k} F>t\right\} \subset$ $\bigcup_{k=0}^{\infty}\left\{M_{a, \gamma, p}^{k} F>t / A 2^{k(a-Q / p) / 2}\right\}$, and using the subadditivity of $H_{\infty}^{m}$, Lemma 
3.3, Lemma 3.4 and Theorem 2.1, we obtain, as we wanted to show, that

$$
\begin{aligned}
\int_{0}^{\infty} & H_{\infty}^{m}\left(\left\{T_{\gamma, \lambda} f>t\right\}\right) t^{p-1} d t \\
\leq & C \int_{0}^{\infty} H_{\infty}^{m}(\{M f>t\}) t^{p-1} d t \\
& +C \sum_{0}^{\infty} \int_{0}^{\infty} H_{\infty}^{m}\left(\left\{M_{a, \gamma, p}^{k} F>t / A 2^{k(a-Q / p) / 2}\right\}\right) t^{p-1} d t \\
\leq & C\|f\|_{p, a}^{p}+C \sum_{0}^{\infty} 2^{k p(a-Q / p) / 2}\|N F\|_{p}^{p} \\
\leq & C\|f\|_{p, a}^{p},
\end{aligned}
$$

where the last inequality is a consequence of the Hardy-Littlewood maximal theorem.

We prove finally Lemmas 3.3 and 3.4.

Proof of Lemma 3.3. Assume $(y, t)$ is such that $t \leq 1$ and $\mathrm{d}(x, y) \leq t^{\gamma}=T$, and set $B^{*}=B(y, 2 T)$; then

$$
\begin{aligned}
|u(y, t)| & \leq\left[\int_{B^{*}}+\int_{X-B^{*}}\right] \Phi_{t}(y, z)|f(z)| d z \\
& =u_{1}(y, t)+u_{2}(y, t) .
\end{aligned}
$$

If $\mathrm{d}(y, z) \geq 2 T$, then $\mathrm{d}(x, z) \leq 3 K \mathrm{~d}(y, z) / 2$ and, as a consequence, $\Phi_{t}(y, z) \leq t^{-Q_{\phi}} \phi(2 \mathrm{~d}(x, z) / 3 K t)$, which under the assumptions on $\phi$ implies by well-known arguments

$$
u_{2}(y, t) \leq t^{-Q} \int_{X} \phi(\mathrm{d}(x, z) / C t)|f(z)| d z \leq C M f(x) .
$$

To deal with $u_{1}(y, t)$, if $B^{\prime}=B(y, t)$, let $B_{0}=B^{\prime} \subset B_{1} \subset \cdots \subset B_{N}=B^{*}$ be a sequence of balls with $B_{i}=B\left(y, 2^{i} t\right)$ and set $R_{0}=B_{0}, R_{i}=B_{i}-B_{i-1}$, $1 \leq i \leq N$. Then,

$$
u_{1}(y, t) \leq t^{-Q} \sum_{0}^{N} \int_{R_{i}}|f(z)| \phi(\mathrm{d}(y, z) / t) d z=\sum_{0}^{N} v_{i}(y, t) .
$$

Since $x \in B_{N}$ and $\phi(\mathrm{d}(y, z) / t) \sim \phi\left(2^{i}\right)$ on $R_{i}$, using the coherence and mean dominance properties and the definition of $f_{a}^{\#}$, we estimate each $v_{i}$ as

$$
\begin{aligned}
& v_{i}(y, t) \leq t^{-Q_{\phi}\left(2^{i}\right)} \int_{R_{i}}\left[\left|f-A_{B_{i}} f\right|+\sum_{i+1}^{N}\left|A_{B_{j}} f-A_{B_{j-1}} f\right|+\left|A_{B_{N}} f\right|\right](z) d z \\
& \leq C 2^{i Q_{\phi}}\left(2^{i}\right)\left[\sum_{i}^{N}\left(2^{j} t\right)^{a} f_{B_{j}} f_{a}^{\#}(z) d z+M f(x)\right] \\
& \left.\leq C 2^{i Q_{\phi}} 2^{i}\right)\left(M f(x)+\sum_{i}^{\infty} 2^{j(a-Q / p)} M_{a, \gamma, p}^{j} F(x)\right),
\end{aligned}
$$


and, therefore,

$$
\begin{aligned}
u_{1}(y, t) & \leq C \sum_{0}^{\infty} 2^{i Q} \phi\left(2^{i}\right)\left(M f(x)+\sum_{i}^{\infty} 2^{j(a-Q / p)} M_{a, \gamma, p}^{j} F(x)\right) \\
& \leq C M f(x)+C \sum_{0}^{\infty} 2^{j(a-Q / p)} M_{a, \gamma, p}^{j} F(x),
\end{aligned}
$$

which together with (4) gives the desired estimate for $u$.

Proof of Lemma 3.4. Set $M_{1} f(x)=\sup \left\{f_{B}|f| d z: x \in B, \mathrm{r}(B)<1\right\}, M_{2} f(x)$ $=\sup \left\{f_{B}|f| d z: x \in B, \mathrm{r}(B) \geq 1\right\}$. If $\left\{B_{k}\right\}=\left\{B\left(x_{k}, r_{k}\right)\right\}$ is a Whitney decomposition of $\left\{M_{2} f>t\right\}$, arguing as in the proof of Lemma 2.3, we see that $\left\{M_{2} f>t\right\} \subset \bigcup\left\{B\left(x_{k}, C r_{k}\right): r_{k}>1\right\}$ and, therefore,

$$
H_{\infty}^{m}\left(\left\{M_{2} f>t\right\}\right) \leq C \sum_{r_{k}>1} r_{k}^{m} \leq C \sum r_{k}^{Q} \leq C\left|\left\{M_{2} f>t\right\}\right| .
$$

Thus, $\left\|M_{2} f\right\|_{L^{p}\left(H_{\infty}^{m}\right)} \leq C\left\|M_{2} f\right\|_{p} \leq C\|f\|_{p}$.

Next, if $x \in B(y, r), r<1$ and $B_{0}=B(y, r) \subset B_{1} \subset \cdots \subset B_{N}=B(y, 1)$ is a sequence of balls with $B_{i}=B\left(y, 2^{i} r\right)$, arguing as in the estimate for the $v_{i}$ above, we obtain

$$
f_{B(y, r)}|f(z)| d z \leq C \sum_{0}^{N}\left(2^{j} r\right)^{a} f_{B_{j}} f_{a}^{\#}(z) d z+C f_{B(y, 1)}|f(z)| d z .
$$

Choosing now $b<a$ such that $\gamma=(Q-b p) / m<1$, it follows that $\left(y, 2^{j} r\right) \in$ $\Omega_{\gamma, \lambda}(x)$ for $0 \leq j \leq N$ and, thus, with $F$ as in Lemma 3.3 and writing $M_{b, \gamma, p}$ for $M_{b, \gamma, p}^{1}$,

$$
\begin{aligned}
f_{B(y, r)}|f(z)| d z & \leq C \sum_{0}^{N}\left(2^{j} r\right)^{a-b}\left(2^{j} r\right)^{b} F\left(y, 2^{j} r\right)+C M_{2} f(x) \\
& \leq C\left(\sum_{0}^{N}\left(2^{j} r\right)^{a-b}\right) M_{b, \gamma, p} F(x)+C M_{2} f(x) \\
& \leq C\left(M_{b, \gamma, p} F(x)+M_{2} f(x)\right) ;
\end{aligned}
$$

the desired result follows now from Theorem 2.1.

\section{TANGENTIAL CONVERGENCE ON STRATIFIED NILPOTENT LIE GROUPS}

Let $\mathbf{N}$ be a simply connected nilpotent Lie group, with identity denoted by 0 , and Lie algebra $\mathbf{n}$, such that

$$
\mathbf{n}=V_{1} \oplus \cdots \oplus V_{m}
$$

with the property $\left[V_{1}, V_{j}\right]=V_{j+1}, 1 \leq j<m,\left[V_{1}, V_{m}\right]=\{0\}$ (we refer to [FoS2, Chapter 1] for unexplained concepts or notation); the exponential exp: $\mathbf{n} \rightarrow \mathbf{N}$ is then a diffeomorphism which takes Lebesgue measure in $\mathbf{n}$ into bi-invariant Haar measure $d x$ in $\mathbf{N}$. We will assume the existence in $\mathbf{n}$ of a family of dilations $\left\{\delta_{r}\right\}_{r>0}$ and denote by $r n$ the induced dilations $r n=$ $\exp \left(\delta_{r}\left(\exp ^{-1}(n)\right)\right)$ on $\mathbf{N}$; we will also assume the existence of a homogeneous 
norm on $\mathbf{N}$, that is, a function $|\cdot|: \mathbf{N} \rightarrow[0, \infty)$ such that $|n|=0$ if and only if $n=0,\left|n^{-1}\right|=|n|$ and $|r n|=r|n|$. It then follows that there is a number $K \geq 1$ such that for all $n, u \in \mathbf{N},|n u| \leq K(|n|+|u|)$ and, therefore, $\mathrm{d}(n, u)=$ $\left|n^{-1} u\right|$ defines a quasidistance on $\mathbf{N}$ whose balls $B(n, r)=\{u: \mathrm{d}(n, u)<r\}$ satisfy $|B(n, r)|=c r^{Q}$ where $c$ is the Haar measure of the unit ball, and $Q=\sum_{j=1}^{m} j \operatorname{dim} V_{j}$ is the homogeneous dimension of $\mathbf{N}$. Fix a basis of left invariant vector fields $\left\{X_{1}, \ldots, X_{n_{1}}, X_{n_{1}+1}, \ldots, X_{n_{2}}, \ldots, X_{n_{m}}\right\}$ in such a way that $\left\{X_{n_{i-1}+1}, \ldots, X_{n_{i}}\right\}$ forms a basis for $V_{i}$ and let $\left\{\xi_{1}, \ldots, \xi_{n}\right\}, n=n_{m}$, be its dual basis. We say that $P: \mathbf{N} \rightarrow \mathbb{C}$ is a polynomial if $P \circ \exp$ is a polynomial on $\mathbf{n}$; setting $\eta_{i}=\xi_{i} \circ \exp ^{-1}$, the functions $\left\{\eta_{1}, \ldots, \eta_{n}\right\}$ form a global coordinate system on $\mathbf{N}$, and any polynomial $P$ can be written as $P=\sum a_{I} \eta^{I}$, where $I=\left(i_{1}, \ldots, i_{n}\right) \in \mathbb{N}^{n}, \eta^{I}=\eta_{1}^{i_{1}}, \ldots, \eta_{n}^{i_{n}}$ and all but finitely many of the $a_{I}$ are 0 . Setting $d_{j}=i$ for $n_{i-1}+1 \leq j \leq n_{i}$ and $d(I)=d_{1} i_{1}+\cdots+d_{n} i_{n}$, the homogeneous degree of such a polynomial $P$ is $\operatorname{deg}(P)=\max \left\{d(I): a_{I} \neq 0\right\}$. The set $\mathbb{P}_{k}$ of polynomials of homogeneous degree $\leq k$ is invariant under dilations and under left and right translations [FoS2, p. 23].

We will denote by $Y_{1}, \ldots, Y_{n}$ the right invariant vector fields in $\mathbf{N}$ such that $\left.X_{i}\right|_{0}=\left.Y_{i}\right|_{0}$, and set $X^{I}=X_{1}^{i_{1}} \cdots X_{n}^{i_{n}}, Y^{I}=Y_{1}^{i_{1}} \cdots Y_{n}^{i_{n}}$, and $(\partial / \partial \eta)^{I}=$ $\partial^{i_{1}} / \partial \eta_{1}^{i_{1}} \cdots \partial^{i_{n}} / \partial \eta_{n}^{i_{n}}$, where the $\partial / \partial \eta_{i}$ are the partial derivatives with respect to the global coordinates $\eta_{i}$. The powers $X^{I}$ and $(\partial / \partial \eta)^{I}$ are related by

$$
X^{I} f=\sum_{\substack{|J| \leq|I| \\ d(J) \geq d(I)}} P_{I J}(\partial / \partial \eta)^{J} f, \quad(\partial / \partial \eta)^{I} f=\sum_{\substack{|J| \leq|I| \\ d(J) \geq d(I)}} Q_{I J} X^{J} f,
$$

where $P_{I J}, Q_{I J}$ are homogeneous polynomials of degree $d(J)-d(I)$ [FoS2, p. 25], and similar formulas hold for $Y^{I}$. As a consequence if $P \in \mathbb{P}_{k}$ then its derivatives $X^{I} P, Y^{I} P \in \mathbb{P}_{k-d(I)}$ for $d(I) \leq k$ and they are equal to 0 if $d(I)>k$. Conversely, if $f \in C^{k}$ and either $X^{I} f=0$ or $Y^{I} f=0$ for all $I$ with $d(I)=k$, it follows from the stratified Taylor inequality [FoS2, p. 34] that $f \in \mathbb{P}_{k-1}$.

If $B$ is a ball in $\mathbf{N}, k \in \mathbb{N}$ and $f \in L_{\text {loc }}^{1}$, let $A_{B}^{k} f$ be the orthogonal projection of $f$ into the vector space $\mathbb{P}_{k}(B)$ of restrictions to $B$ of polynomials of degree $\leq k$ endowed with the inner product $(\phi, \psi)=|B|^{-1} \int_{B} \phi \psi$. We have

Proposition 4.1. For each $k, A^{k}=\left\{A_{B}^{k}\right\}$ is a family of averaging operators. Moreover, for any ball $B$,

$$
\int_{B}\left|f-A_{B}^{k} f\right| \simeq \inf _{P \in \mathbb{P}_{k}} \int_{B}|f-P| .
$$

Proof. The linearity and coherence properties are obvious. An easy homogeneity argument yields, for $|u|<1$,

$$
A_{B(0,1)}^{k}\left[f \circ L_{n} \circ r\right](u)=A_{B(n, r)}^{k} f(n r u),
$$

where $L_{n}$ denotes left translation by $n$; thus the mean dominance for the ball $B(n, r)$ reduces to that for $B(0,1)$, which follows by writing $A_{B(0,1)}^{k} f$ in terms of an orthonormal basis of $\mathbb{P}_{k}(B(0,1))$. Finally, estimate $(5)$ is an easy consequence of the coherence and mean dominance properties. 
Given $a>0$ and $k=[a]$ we will denote by $C^{p, a}$ the space $C_{A_{k}}^{p, a}$. For $1 \leq p<\infty$ and $k \in \mathbb{N}$ we consider the Sobolev spaces

$$
W^{p, k}=\left\{f \in L^{p}: X^{I} f \in L^{p} \text { for } d(I) \leq k\right\}
$$

with the norm $\|f\|_{W^{p, k}}=\sum_{d(I) \leq k}\left\|X^{I} f\right\|_{p}$. The main result of this section is

Theorem 4.2. If $1<p<\infty, k \in \mathbb{N}$ and $f \in W^{p, k}$, then $f \in C^{p, k}$ and $\|f\|_{p, k} \leq C\|f\|_{W^{p, k}}$.

Since $C_{0}^{\infty}$ is dense in $W^{p, k}$ [Fol, p. 187] we have

Corollary 4.3. If $Q-k p<m \leq Q, \gamma=(Q-k p) / m, f \in W^{p, k}, \phi(r)$ and $u(n, t)$ are as in Theorem 3.2 and we assume $\int \phi(|n|) d n=1$, then $u(n, t)$ tends to $f(n)$ inside $\Omega_{\gamma, \lambda}(n)$ for $H^{m}$-a.e. $n \in \mathbf{N}$ and all $\lambda>0$.

We remark that these two results also hold for the more general Bessel potential spaces $J_{a}\left(L^{p}\right)$ of G. B. Folland (see their definition below).

Before continuing, we shall give three specific examples on tangential convergence for harmonic and holomorphic functions that follow directly from the Corollary 4.3. All three involve the class $\mathscr{R}$ [FoS2, p. 253] of those $\phi \in C^{\infty}(\mathbf{N})$ such that $\Phi(n, t)=t^{-Q} \phi(n / t)$ for $t \neq 0$, and $\Phi(n, 0)=0$ for $n \neq 0$, defines a $C^{\infty}$ function on $\mathbf{N} \times[0, \infty)-\{(0,0)\}$. Such a $\phi$ satisfies $|\phi(n)| \leq C /\left(1+|n|^{Q+1}\right)[\mathrm{FoS} 2$, p. 255] and the preceding tangential convergence results apply to

$$
u(n, t)=(f * \Phi(\cdot, t))(n)=\int f\left(n u^{-1}\right) \Phi(u, t) d u
$$

Example 1. Our first and more general example deals with the Poisson kernel $P(n, t)$ associated to the sublaplacian $\mathscr{L}=\sum_{i=1}^{n_{1}} X_{i}^{2}$. For any bounded continuous function $f$ defined on $\mathbf{N}$, the solution to the Dirichlet problem $\left(\mathscr{L}+\partial^{2} / \partial t^{2}\right) u=0, u(n, 0)=f(n)$ is given (cf. [Fo2]) by

$$
u(n, t)=[f * P(\cdot, t)](n),
$$

where $P(n, t)=t^{-Q} p(n / t)$ is the Poisson kernel for $\mathbf{N}$ and $p \in \mathscr{R}$.

Example 2. Let $\mathbf{X}=G / K$ be a rank one symmetric space of noncompact type, where $G=K A \mathbf{N}$ is the Iwasawa decomposition of the semisimple Lie group $G$ and let $\overline{\mathbf{N}}$ be the image of $\mathbf{N}$ under the Cartan involution. The nilpotent Lie groups $\mathbf{N}$ and $\overline{\mathbf{N}}$ are either step one or step two nilpotent (see [He, Chapter IX] for details). Choose a vector $H$ in the Lie algebra of $A$ such that $r n=$ $\exp (t H) n \exp (-t H)$, where $t=\log (1 / r)$, defines a family of dilations in $\overline{\mathbf{N}}$. If $\mathbf{n}=V_{1} \oplus V_{2}$ and $\left\{X_{1}, \ldots, X_{p}\right\}$ is a basis of $V_{1}$ and $\left\{Y_{1}, \ldots, Y_{q}\right\}$ is a basis of $V_{2}$ (when needed), for each $n=\exp \left\{\sum_{1}^{p} x_{i} X_{i}+\sum_{1}^{q} y_{j} Y_{j}\right\}$ in $\overline{\mathbf{N}}$ we take as coordinates $(x, y)=\left(x_{1}, \ldots, x_{p}, y_{1}, \ldots, y_{q}\right)$ and as homogeneous norm $|n|=\left(16|y|^{2}+|x|^{4}\right)^{1 / 4}$. If $\Delta_{\mathbf{X}}$ denotes the Laplace-Beltrami operator on $\mathbf{X}$, for any bounded continuous function $f$ on $\overline{\mathbf{N}}$, the solution to the Dirichlet problem $\Delta \mathbf{x} u=0$ in $\mathbf{X},\left.u\right|_{\overline{\mathbf{N}}}=f$, is given by $u(n \exp (t H) K)=$ $\int f\left(n u^{-1}\right) r^{-Q} \phi\left(r^{-1} u\right) d u$, where now $Q=p+2 q$ and

$$
\phi(n)=C\left[\left(1+c|x|^{2}\right)^{2}+4 c|y|^{2}\right]^{-Q / 2} \in \mathscr{R} .
$$


Example 3 (the Siegel domains). Let

$$
D=\left\{z=\left(z^{\prime}, z_{n+1}\right): z^{\prime} \in \mathbb{C}^{n}, z_{n+1} \in \mathbb{C}, h(z)=\operatorname{Im} z_{n+1}-\left|z^{\prime}\right|^{2}>0\right\}
$$

and let $\partial D$ be its houndary. We identify $\partial D$ with the Heisenberg group $\mathbb{H}$ that can be parametrized by $\mathbb{C}^{n} \times \mathbb{R}$ with the operation $\left(z^{\prime}, t\right)\left(w^{\prime}, s\right)=\left(z^{\prime}+\right.$ $\left.w^{\prime}, t+s+2 z^{\prime} \cdot \overline{w^{\prime}}\right)$. The group $\mathbb{H}$ is step two nilpotent with dilations defined by $r\left(z^{\prime}, t\right)=\left(r z^{\prime}, r^{2} t\right)$ and its homogeneous norm is $\left|\left(z^{\prime}, t\right)\right|=\left(t^{2}+\left|z^{\prime}\right|^{4}\right)^{1 / 4}$. For $f$ bounded and continuous on $\mathbb{H}$ the solution to the Dirichlet problem $\Delta_{D} u=0,\left.u\right|_{\partial D}=f$ is given by the function

$$
P f\left(z^{\prime}, t+i\left(\tau+\left|z^{\prime}\right|^{2}\right)\right)=\int_{\mathbb{H}} f\left(\left(z^{\prime}, t\right) w^{-1}\right) P_{\tau}(w) d w
$$

where $P_{\tau}(w)=\tau^{-Q} P\left(\tau^{-1} w\right), Q=2 n+2$ and

$$
P\left(z^{\prime}, t\right)=c /\left(t^{2}+\left(1+\left|z^{\prime}\right|^{2}\right)^{2}\right)^{n+1} \in \mathscr{R} .
$$

We also consider the Cauchy-Szegö kernel in $D$ :

$$
S(z, w)=c\left(i\left(\overline{w_{n+1}}-z_{n+1}\right)-2 z^{\prime} \cdot \overline{w^{\prime}}\right)^{-n} \quad(z \in D, w \in \partial D) .
$$

If $f \in L^{p}(\mathbb{H}), 1<p<\infty$, the holomorphic function defined by the CauchySzegö integral $F(z)=\int_{\mathbb{H}} S(z, w) f(w) d w$ can be expressed as $F(z)=$ $P\left(\frac{1}{2} f+S f\right)(z)[\mathrm{KV}$, p. 618] where $S$ is a principal value distribution and, hence, bounded in $W^{p, k}$ [FoS1, p. 455]. Therefore, tangential convergence is also obtained for Cauchy-Szegö integrals of Sobolev functions.

Before sketching the proof of Theorem 4.2 we first recall Folland's definition of Bessel potentials on the group $\mathbf{N}$ [Fol]. Let $h(n, t), n \in \mathbf{N}, t>0$ be the heat kernel associated to the heat operator $\partial / \partial t-\sum_{i=1}^{n_{1}} X_{i}^{2}$ and, for $a>0$, set

$$
J_{a}(n)=\frac{1}{\Gamma(a / 2)} \int_{0}^{\infty} t^{a / 2} e^{-t} h(n, t) d t / t
$$

The following lemma gives the behavior of $J_{a}(n)$ for $|n|$ near 0 or $\infty$.

Lemma 4.4. If $a<Q$ and $n \neq 0$ then

$$
\left|X^{I} J_{a}(n)\right| \leq C|n|^{a-Q-d(I)} \quad\left(I \in \mathbb{N}^{n}\right) ;
$$

moreover, for all $k \in \mathbb{N}$, there exists $C=C_{I, k}$ such that

$$
\left|X^{I} J_{a}(n)\right| \leq C|n|^{-Q-d(I)-2 k} \text {. }
$$

Proof. Differentiating under the integral sign the expression defining $J_{a}$ and taking into account that for all $k$ and $I$ the inequality $\left|\partial_{t}^{k} X^{I} h(n, t)\right| \leq$ $C(\sqrt{t}+|n|)^{-Q-d(I)-2 k}$ holds [FoS2], we obtain

$$
\begin{aligned}
\left|X^{I} J_{a}(n)\right| \leq & C|n|^{-Q-d(I)} \int_{0}^{|n|^{2}} t^{a / 2} e^{-t} \frac{d t}{t} \\
& +C \int_{|n|^{2}}^{\infty} t^{(a-Q-d(I)) / 2} e^{-t} \frac{d t}{t} \\
\leq & C|n|^{a-Q-d(I)} .
\end{aligned}
$$

Next, since for $n \neq 0$ the function $X^{I} h(n, t)$ vanishes to infinite order at $t=0$, Taylor's theorem yields $\left|X^{I} h(n, t)\right| \leq C t^{k}|n|^{-Q-d(I)-2 k}$ for all $k$, and the second estimate also follows by differentiating under the integral sign. 
Our interest in the Bessel kernels is due to the fact that $W^{p, k}=J_{k} L^{p}$ (cf. [Fol, p. 191]). Since $J_{b}\left(C^{p, a}\right)$ is continuously imbedded in $C^{p, a+b}$ (see Proposition 4.5 below), we have that $W^{p, k}=J_{k}\left(L^{p}\right)=J_{k}\left(C^{p, 0}\right) \subset C^{p, k}$, and then Theorem 4.2 follows.

Proposition 4.5. If $f \in C^{p, a}, a \geq 0$, then $\left(J_{b} f\right)_{a+b}^{\#} \leq C f_{a}^{\#}$ and therefore $\left\|J_{b} f\right\|_{a+b, p} \leq\|f\|_{a, p}$.

Proof. We simply sketch the argument. We assume $b<Q$ (the general case will follow from the semigroup property of Bessel potentials, cf. [Fo1, p. 183]). We fix $n \in \mathbf{N}$ and $r>0$, and set $k=[a]$, and $l=[a+b]$, where [ $\cdot]$ denotes as before the integral part function. Let $B=B(n, r)$ and $B^{\prime}=B(n, 2 K \lambda r)$, where $\lambda$ and $K$ denote the constant in the stratified left Taylor inequality (cf. [FoS2, p. 35]) and the constant in the quasidistance inequality for $\mathbf{N}$ respectively. Let $P=A_{B^{\prime}}^{k} f$ and

$$
\begin{aligned}
Q(u) & =P * J_{b}(u)+\int_{c^{\prime}}(f(v)-P(v)) T_{v^{-1} n}^{l} J_{b}\left(n^{-1} u\right) d v \\
& =Q_{1}(u)+Q_{2}(u),
\end{aligned}
$$

where $T_{v^{-1} n}^{l}(\cdot)=T(\cdot)$ stands for the degree $l$ left Taylor polynomial of $J_{b}$ at the point $v^{-1} n \in \mathbf{N}$. By Lemma 4.4, $Q_{1}$ is well defined and since $d(I)=k+1$ implies $Y^{I} Q_{1}=\left(Y^{I} P\right) * J_{b}=0$, we get $Q_{1} \in \mathbb{P}_{k}$. On the other hand,

$$
\begin{aligned}
T\left(n^{-1} u\right) & =\sum_{d(I) \leq l} c_{I}(\partial / \partial \eta)^{I} T(0) \eta\left(n^{-1} u\right)^{I} \\
& =\sum_{d(I)<l}\left\{\sum_{\substack{|J| \leq|I| \\
d(J)=d(I)}} c_{I, J} X^{J} T(0)\right\} \eta\left(n^{-1} u\right)^{I} \\
& =\sum_{d(I) \leq l}\left\{\sum_{\substack{|J| \leq|I| \\
d(J)=d(I)}} c_{I, J} X^{J} J_{b}\left(v^{-1} n\right)\right\} \eta\left(n^{-1} u\right)^{I}
\end{aligned}
$$

which, again by Lemma 4.4 , implies that $Q_{2}$ is well defined and belongs to $\mathbb{P}_{l}$. Now

$$
\begin{aligned}
f_{B}\left|J_{b} f-Q\right| d u \leq & f_{B}\left(\int_{B^{\prime}}|f(v)-P(v)| J_{b}\left(v^{-1} u\right) d v\right) d u \\
& +f_{B}\left(\int_{c^{\prime}}|f(v)-P(v)|\left|J_{b}\left(v^{-1} u\right)-T\left(n^{-1} u\right)\right| d v\right) d u \\
& =\mathrm{I}+f_{B} \mathrm{II}(u) d u
\end{aligned}
$$

and arguing as in [Do, p. 675], it follows that both $\mathrm{I}$ and $\mathrm{II}(u)$ are bounded by $C r^{a+b} f_{a}^{\#}(n)$, which implies the desired result.

\section{HARMONIC FUNCTIONS IN LIPSCHITZ DOMAINS}

A bounded domain $D \subset \mathbb{R}^{N+1}$ is called a Lipschitz domain if for each $Q \in \partial D$ there is a ball $B^{\prime}=B\left(Q, \rho_{0}\right)$, a coordinate system $\left(x, x_{N+1}\right)=$ 
$\left(x_{1}, \ldots, x_{N+1}\right)$ isometric to the usual one in $\mathbb{R}^{N+1}$ and a function $\phi$ with $\|\nabla \phi\|_{\infty} \leq m$ such that $B^{\prime} \cap D=\left\{\left(x, x_{N+1}\right): x_{N+1}>\phi(x)\right\} \cap D$. We shall assume the constants $\rho_{0}$ and $m$ to be the same for all $Q \in \partial D$ and set $\Phi(x)=(x, \phi(x))$ and $\Delta(Q, r)=B(Q, r) \cap \partial . D$.

Let us briefly discuss Dirichlet's problem in Lipschitz domains (see [Dal or $\mathrm{JK}$ ] for more details). If $f$ is continuous on $\partial D$, the function $u \in C^{2}(D) \cap$ $C(\bar{D})$ such that $\Delta u=0$ on $D, u=f$ on $\partial D$ is given by

$$
u(X)=\int_{\partial D} f(Q) d \omega^{X}(Q),
$$

where $d \omega^{X}$ denotes harmonic measure with respect to $D$ and $X$. By Harnack's inequality, for all $X, Y \in D, d \omega^{X} \ll d \omega^{Y}$; therefore, fixing a point $X_{0} \in D$ and setting $d \omega=d \omega^{X_{0}}$, it follows that for $X \in D$ and $Q \in \partial D, d \omega^{X}(Q)=$ $K(X, Q) d \omega(Q)$.

$K(X, Q)$ is called the kernel function of $D$, and it has [JK, Lemma 4.14] the following property: there is a sequence $\left\{c_{j}\right\}$ with $\sum_{0}^{\infty} c_{j}<\infty$ verifying that for any $Q_{0} \in \partial D$ and any $r, 0<r<\rho_{0}$, a point $A=A_{r}\left(Q_{0}\right)$ can be found in $D$ with $\left|A-Q_{0}\right| \simeq r$ such that setting $\Delta_{0}=\Delta\left(Q_{0}, r\right)=R_{0}, \Delta_{j}=\Delta\left(Q_{0}, 2^{j} r\right)$, and $R_{j}=\Delta_{j}-\Delta_{j-1}$, then for all $Q \in R_{j}$,

$$
K(A, Q) \leq c_{j} / \omega\left(\Delta_{j}\right) .
$$

Furthermore, $d \omega$ and surface measure $d \sigma$ are also mutually absolutely continuous and, for a certain $q>1$, the Radon-Nikodym derivative $k=d \omega / d \sigma$ verifies the following reverse Hölder condition [Da1, Theorem 3]: for every $\Delta \subset \partial D$,

$$
\left(f_{\Delta} k^{q} d \sigma\right)^{1 / q} \leq C f_{\Delta} k d \sigma
$$

$q$ can always be taken $>2$ for general Lipschitz domains, and for $C^{1}$ domains (7) holds for all $q<\infty$.

We will also need the following quantitative form of the already mentioned Harnack's inequality [JK, p. 93]: if $X_{1}, X_{2} \in D$ verify

$$
\delta=\delta\left(X_{1}\right)=\operatorname{dist}\left(X_{1}, \partial D\right) \simeq \delta\left(X_{2}\right)
$$

and $\left|X_{1}-X_{2}\right| \leq \delta$, then, for any positive harmonic function $u, u\left(X_{1}\right) \simeq u\left(X_{2}\right)$.

We introduce now Sobolev spaces $W^{p, 1}(\partial D)$. Fix first a finite family $\left\{B_{i}^{\prime}=\right.$ $\left.B\left(Q_{i}, \rho_{0}\right)\right\}$ such that $\partial D=\bigcup \Delta_{i}^{*}$, with $\Delta_{i}^{*}=\Delta\left(Q_{i}, \rho_{0} / 4 M\right), M=\sqrt{1+m^{2}}$, and denote the corresponding coordinate functions as $\phi_{i}, \Phi_{i}$; then $f$ will be in $W^{p, 1}(\partial D)$ if and only if $f \in L^{p}(d \sigma)$ and for each $i, f_{i}(x)=f\left(\Phi_{i}(x)\right) \in$ $W^{p, 1}\left(\Phi_{i}^{-1}\left(\Delta_{i}^{\prime}\right)\right)$, where $\Delta_{i}^{\prime}=B_{i}^{\prime} \cap \partial D$ (the Lipschitz character of $\partial D$ makes the definition independent of the choice of the $\left.B_{i}^{\prime}\right)$. We also define $C^{p, 1}(\partial D)$ as the space of those $f \in L^{p}(d \sigma)$ such that $f_{1}^{\#} \in L^{p}(d \sigma)$, where

$$
f_{1}^{\#}\left(Q_{0}\right)=\sup _{Q_{0} \in \Delta} \mathrm{r}(\Delta)^{-1} f_{\Delta}\left|f(Q)-f_{\Delta}\right| d \sigma(Q),
$$

with $f_{\Delta}=f_{\Delta} f$. We have 
Lemma 5.1. If $f \in W^{p, 1}, 1<p<\infty$, then $f_{1}^{\#} \in L^{p}(d \sigma)$.

Proof. Let $Q_{0} \in \Delta(Q, r)=\Delta$; we will assume that $r(\Delta)<\rho_{0} / 4 M$, since for $r \geq \rho_{0} / 4 M, r^{-1} f_{\Delta}\left|f-f_{\Delta}\right| d \sigma \leq C M f\left(Q_{0}\right)$, with $M$ denoting HardyLittlewood's maximal operator with respect to surface measure. If $Q_{0} \in \Delta_{i}^{*}$, then $\Phi^{-1}(\Delta) \subset \mathscr{B}=B\left(\Phi_{i}^{-1}(Q), r\right) \subset \Phi_{i}^{-1}\left(\Delta_{i}^{\prime}\right) \subset \mathbb{R}^{N}$ and, therefore, setting $f_{\mathscr{B}}=f_{\mathscr{B}} f_{i}(p) d p$, Poincaré's inequality in $\mathbb{R}^{N}$ gives us

$$
\begin{aligned}
f_{\Delta}\left|f-f_{\Delta}\right| d \sigma & \leq C f_{\Delta}\left|f-f_{\mathscr{B}}\right| d \sigma \\
& \leq C f_{\mathscr{B}}\left|f_{i}(p)-f_{\mathscr{B}}\right| d p \leq C r F_{i}\left(Q_{0}\right)
\end{aligned}
$$

where $F_{i}\left(Q_{0}\right)=\sup \left\{f_{\mathscr{B}(q, t)}\left|\nabla f_{i}(p)\right| d p:\left|Q_{0}-\Phi_{i}(q)\right| \leq t \leq \rho_{0} / 4 M\right\}$. Clearly $F_{i} \in L^{p}\left(\Delta_{i}^{\prime}, d \sigma\right)$, and since $f_{1}^{\#} \leq C \sum F_{i}$, we obtain $f_{1}^{\#} \in L^{p}(d \sigma)$.

We observe that since [DVS, Chapter 5] $\left|\nabla f_{i}(p)\right| \leq\left(f_{i}\right)_{1}^{*}(p) \leq f_{1}^{\#}\left(\Phi_{i}(p)\right)$, then $\sum F_{i} \leq C M\left(f_{1}^{\#}\right)$, and as a consequence, $C^{p, 1}=W^{p, 1}$ and

$$
\sum_{1}^{M}\left\|\nabla f_{i}\right\|_{L^{p}\left(\mathscr{R}_{i}^{*}\right)} \simeq\left\|f_{1}^{\#}\right\|_{p} .
$$

For $\lambda>1,0<\gamma<1$, set $\Omega_{\gamma, \lambda}\left(Q_{0}\right)=\left\{X \in D:\left|X-Q_{0}\right| \leq(1+\lambda) \delta(X)^{\gamma}\right\}$. We have the following result on tangential convergence of solutions of the Dirichlet problem with Sobolev functions as boundary data.

Theorem 5.2. Let $D$ be a Lipschitz domain for which $k=d \omega / d \sigma$ satisfies a reverse Hölder condition with exponent $q$. If $f \in W^{p, 1}(\partial D), p<N, q^{\prime} \leq p^{*}=$ $N p /(N-p)$ and $1-p / N \leq \gamma<1$, then $u(X)=\int f(Q) d \omega^{X}(Q)$ tends to $f\left(Q_{0}\right)$ as $X$ tends to $Q_{0}$ inside $\Omega_{\gamma, \lambda}\left(Q_{0}\right)$ for $H^{m}$-a.a. $Q_{0} \in \partial D, m=(N-p) / \gamma$, and all $\lambda>0$.

Proof. Assume $X \in D$ is such that $\left|X-Q_{0}\right| \leq \delta^{\gamma}=\delta(X)^{\gamma}<1$, set $T=\delta^{\gamma}$ and fix $Q_{X} \in \partial D$ with $\left|X-Q_{X}\right|=\delta$. If $\Delta^{*}=\Delta\left(Q_{X}, 2 T\right), A=A_{\delta}\left(Q_{X}\right)$ and $U(X)=\int|f(Q)| d \omega^{X}(Q)$, the above form of Harnack's inequality gives

$$
\begin{aligned}
|u(X)| & \leq C U(A)=C\left(\int_{\Delta^{*}}+\int_{\partial D-\Delta^{*}}\right)|f(Q)| K(A, Q) d \omega(Q) \\
& =C\left(U_{1}(A)+U_{2}(A)\right) .
\end{aligned}
$$

Arguing as in [CFMS, Theorem 4.3] we obtain $U_{2}(A) \leq C M_{\omega} f\left(Q_{0}\right)$, with $M_{\omega} f(Q)=\sup \left\{\int_{\Delta} f d \omega / \omega(\Delta): Q \in \Delta\right\}$. Now, by the well-known self-improving property of reverse Hölder conditions, $k$ does satisfy a condition with exponent $r>q$ and, therefore, $M_{\omega} f \leq C\left(M|f|^{r^{\prime}}\right)^{1 / r^{\prime}}$, where we have now $r^{\prime}<p^{*}$. Using Proposition 3.1 we conclude that $U_{2}(A) \leq C M f\left(Q_{0}\right)+C\left(M_{1, \gamma, p}^{1}(F)\left(Q_{0}\right)\right)$, with $F(y, t)=\left(f_{B(y, t)} f_{1}^{\#)^{\prime}}\right)^{1 / s}$ and $s=N r^{\prime} /\left(N+r^{\prime}\right)<p$.

To deal with $U_{1}(A)$ let $\Delta_{0}=\Delta\left(Q_{X}, \delta\right) \subset \Delta_{1} \subset \cdots \subset \Delta_{M}=\Delta^{*}$ be a sequence of surface balls as in the proof of Lemma 3.4 and set $R_{0}=\Delta_{0}, R_{i}=\Delta_{i}-\Delta_{i-1}$. Using estimate (6) and the reverse Hölder condition and arguing as in that 
lemma, we have for $v_{i}(A)=\int_{R_{i}}|f(Q)| K(A, Q) k(Q) d \sigma(Q)$,

$$
\begin{aligned}
v_{i}(A) \leq & \frac{c_{i}}{\omega\left(\Delta_{i}\right)}\left(\int_{\Delta_{i}}\left|f(Q)-f_{\Delta_{i}}\right| k(Q) d \sigma(Q)\right) \\
& +c_{i}\left(\sum_{j=i+1}^{M} f_{\Delta_{j}}\left|f-f_{\Delta_{j}}\right| d \sigma\right)+c_{i} M f\left(Q_{0}\right) \\
\leq & c_{i} \sum_{j=i}^{M}\left(f_{\Delta_{j}}\left|f-f_{\Delta_{j}}\right|^{r^{\prime}} d \sigma\right)^{1 / r^{\prime}}+c_{i} M f\left(Q_{0}\right) \\
\leq & c_{i} \sum_{j=i}^{M} 2^{j} \delta\left(f_{\Delta_{j}}\left(f_{1}^{\#}\right)^{s} d \sigma\right)^{1 / s}+c_{i} M f\left(Q_{0}\right),
\end{aligned}
$$

where the last inequality follows again from Proposition 3.1 with

$$
s=N r^{\prime} /\left(N+r^{\prime}\right)<p .
$$

Thus, with $F(Q, t)$ as before, we obtain the estimate

$$
|u(X)| \leq C M f\left(Q_{0}\right)+C \sum_{0}^{\infty} 2^{j(1-N / p)} M_{1, \gamma, p}^{j} F\left(Q_{0}\right),
$$

which, arguing as in Theorem 3.2 yields the strong-type inequality

$$
\int_{0}^{\infty} H_{\infty}^{m}\left(\left\{T_{\gamma, \lambda} f>t\right\}\right) t^{p-1} d t \leq C\|f\|_{p, 1}^{p},
$$

with $T_{\gamma, \lambda} f(Q)=\sup \left\{|u(X)|: X \in \Omega_{\gamma, \lambda}(Q)\right\}$. A well-known density argument completes the proof.

We finally remark that the above arguments rely only on the estimate (6) for the kernel function, the reverse Hölder condition (7) and the quantitative version of Harnack inequality stated at the beginning of this section. These three properties are satisfied not only by the Laplacian but also by more general second order elliptic operators in divergence form (see [FJK, Da2]) and the results of this section hold for these operators too. There are corresponding results for higher order Sobolev spaces $W^{p, l}$ in $C^{k}$ domains, $l \leq k$, as well.

\section{FURTHER RESUltS}

We will discuss in this section tangential convergence for functions in $C^{p, a}$ when $a p=Q$ and also nontangential convergence for general $a, p$.

In the first case we will prove a weak-type inequality for the tangential maximal operators associated with certain exponential regions. More precisely, with $\mathbf{X}$ a space of homogeneous type as in $\S 2$, set for $x \in \mathbf{X}$,

$$
\boldsymbol{\Theta}_{\gamma, \lambda}(x)=\left\{(y, t): \mathrm{d}(x, y) \leq(1+\lambda) /(\log 1 / t)^{1 / \gamma}\right\},
$$

let $\phi, \Phi$ and $u$ be functions of the type considered in $\S 3$, and consider the operator

$$
E_{\gamma, \lambda} f(x)=\sup \left\{|u(y, t)|:(y, t) \in \Theta_{\gamma, \lambda}(x)\right\}
$$

we have 
Theorem 6.1. If $f \in C^{p, Q / p}(\mathbf{X})$ and $0<\gamma \leq Q p^{\prime} / p$, then, for all $\lambda>0$,

$$
H_{\infty}^{\gamma p / p^{\prime}}\left(\left\{E_{\gamma, \lambda} f>t\right\}\right) \leq C\left(\|f\|_{p, Q / p} / t\right)^{p} .
$$

A similar result holds for functions in $W^{N, 1}(\partial D)$ with $D \subset \mathbb{R}^{N+1}$ a bounded Lipschitz domain: setting

$$
\Theta_{\gamma, \lambda}\left(Q_{0}\right)=\left\{X \in D:\left|X-Q_{0}\right| \leq(1+\lambda) /(\log 1 / \delta(X))^{1 / \gamma}\right\},
$$

we have for $u(X)=\int f(Q) d \omega^{X}(Q)$ and

$$
E_{\gamma, \lambda} f\left(Q_{0}\right)=\sup \left\{|u(X)|: X \in \Theta_{\gamma, \lambda}\left(Q_{0}\right)\right\},
$$

Theorem 6.2. If $f \in W^{N, 1}(\partial D)$ and $0<\gamma \leq N^{\prime}$, then, for all $\lambda>0$,

$$
H_{\infty}^{\gamma N / N^{\prime}}\left(\left\{E_{\gamma, \lambda} f>t\right\}\right) \leq C\left(\left(\|f\|_{N}+\left\|f_{1}^{\#}\right\|_{N}\right) / t\right)^{N} .
$$

The proof is similar in both cases and, being slightly more complicated, we will only give that of Theorem 6.2.

Proof of Theorem 6.2. Since $D$ is Lipschitz we can assume that harmonic measure $d \omega=d \omega^{X_{0}}$ with respect to the fixed point $X_{0}$ satisfies a reverse Hölder condition with exponent 2 .

Let us consider the function $\Phi(t)=e^{\beta t^{N^{\prime}} / 2}-1$, with $\beta$ the constant of estimate (3) in Proposition 3.1 and $N^{\prime}=N /(N-1)$. Denoting by $\Psi$ its complementary Young function $[Z$, p. 16], then, for some constant $C, \Psi(s) \leq$ $C s\left(1+\log ^{+} s\right)^{1 / N^{\prime}}=\Psi^{\prime}(s)$.

Assume $Q_{0} \in \partial D, X \in D$ and $\left|X-Q_{0}\right| \leq(1+\lambda) / \log (1 / \delta(X))^{1 / \gamma}=\rho$; if $\delta=\delta(X) \leq 1 / 10$ and $Q_{X} \in D$ is such that $\left|X-Q_{X}\right|=\delta$, then $Q_{0} \in$ $\Delta\left(Q_{X}, 2 \rho\right)=\Delta^{*}$, and setting $A=A_{\delta}\left(Q_{X}\right)$, we have as in the proof of Theorem 5.2 ,

$$
\begin{aligned}
|u(X)| & \leq C|U(A)| \leq C\left(\int_{\Delta^{*}}+\int_{\partial D-\Delta^{*}}\right)|f(Q)| d \omega^{A}(Q) \\
& \leq C M_{\omega} f\left(Q_{0}\right)+C \int_{\Delta^{*}}\left|f(Q)-f_{\Delta^{*}}\right| d \omega^{A}(Q)+M f\left(Q_{0}\right) \\
& \leq C\left(M f^{2}\left(Q_{0}\right)\right)^{1 / 2}+C \int_{\Delta^{*}}\left|f(Q)-f_{\Delta^{*}}\right| d \omega^{A}(Q) \\
& =C\left(M f^{2}\left(Q_{0}\right)\right)^{1 / 2}+C V(A) .
\end{aligned}
$$

Set now $F(Q)=\left|f(Q)-f_{\Delta^{*}}\right| /\left\|f_{1}^{\#} \chi_{\Delta^{*}}\right\|_{N}$; then, by Hölder's inequality for the pair $\Phi, \Psi[Z$, p. 175],

$$
\begin{aligned}
V(A)= & \omega\left(\Delta^{*}\right)\left\|f_{1}^{\#} \chi_{\Delta^{*}}\right\|_{N} \int_{\Delta^{*}} F(Q) K(A, Q) \frac{d \omega(Q)}{\omega\left(\Delta^{*}\right)} \\
\leq & \omega\left(\Delta^{*}\right)\left\|f_{1}^{\#} \chi_{\Delta^{*}}\right\|_{N}\|K(A, \cdot)\|_{\Psi, d \omega(Q) / \omega\left(\Delta^{*}\right)} \\
& \cdot\left(f_{\Delta^{*}} \mathrm{e}^{\beta|F(Q)|^{N^{\prime}} / 2} k(Q) d \sigma(Q)\right)\left(\left|\Delta^{*}\right| / \omega\left(\Delta^{*}\right)\right) \\
\leq & C \omega\left(\Delta^{*}\right)\left\|f_{1}^{\#} \chi_{\Delta^{*}}\right\|_{N}\|K(A, \cdot)\|_{\Psi, d \omega(Q) / \omega\left(\Delta^{*}\right)} \\
& \cdot\left(f_{\Delta^{*}} e^{\beta|F(Q)|^{N^{\prime}}} d \sigma(Q)\right)^{1 / 2}\left(f_{\Delta^{*}} k^{2} d \sigma\right)^{1 / 2}\left(|\Delta|^{*} / \omega\left(\Delta^{*}\right)\right) \\
\leq & C \omega\left(\Delta^{*}\right)\left\|f_{1}^{\#} \chi_{\Delta^{*}}\right\|_{N}\|K(A, \cdot)\|_{\Psi, d \omega(Q) / \omega\left(\Delta^{*}\right)},
\end{aligned}
$$


the last estimate being a consequence of the second part of Proposition 3.1 and the reverse Hölder condition satisfied by $k$.

We estimate $\|K(A, \cdot)\|_{\Psi, d \omega(Q) / \omega\left(\Delta^{*}\right)}$. Set $\Delta=\Delta\left(Q_{X}, \delta\right)$ and

$$
T=c_{0}\left(\log (e / \omega(\Delta))^{1 / N^{\prime}} / \omega\left(\Delta^{*}\right),\right.
$$

with $c_{0} \geq 1$ to be fixed later. Since $\omega\left(\Delta^{\prime}\right) \leq 1$ for all surface balls, we have $T \geq 1$; also, $k$ verifies for some $p$ Muckenhoupt's $A_{p}$ condition [CF],

$$
\left(f_{\Delta^{\prime}} k d \sigma\right)\left(f_{\Delta^{\prime}} k^{-1 / p-1}\right)^{p-1} \leq C
$$

and, therefore, $1 / \omega\left(\Delta^{\prime}\right) \leq C /\left|\Delta^{\prime}\right|^{p}$; thus, $T \leq C(\log 1 / \delta)^{N^{\prime}} / \omega\left(\Delta^{*}\right)$. By estimate (6) we have $K(A, Q) \leq c / \omega(\Delta) \leq C \delta^{-N p}$ for all $Q \in \Delta^{*}$, which implies

$$
\begin{aligned}
& \frac{1}{\omega\left(\Delta^{*}\right)} \int_{\Delta^{*}} \Psi(K(A, Q) / T) d \omega(Q) \\
& \quad \leq \frac{C_{\Psi}}{\omega\left(\Delta^{*}\right)} \int_{\Delta^{*}} \frac{K(A, Q)}{T}\left(1+\log ^{+} \frac{K(A, Q)}{T}\right)^{1 / N^{\prime}} d \omega(Q) \\
& \quad \leq \frac{C}{\omega\left(\Delta^{*}\right)} \int_{\Delta^{*}} \frac{K(A, Q)}{T}\left(\log \frac{\mathrm{e}}{\omega(\Delta)}\right)^{1 / N^{\prime}} d \omega(Q) \\
& \quad \leq \frac{C^{\prime}}{c_{0}} \int_{\Delta^{*}} K(A, Q) d \omega(Q) \leq \frac{C}{c_{0}} \leq \Psi(1)
\end{aligned}
$$

for an appropriate $c_{0}$. Since [Z, p. 173],

$$
\|g\|_{\Psi, d \omega / \omega\left(\Delta^{*}\right)}=\inf \left\{t: \int_{\Delta^{*}} \Psi(g / t) d \omega / \omega\left(\Delta^{*}\right) \leq \Psi(1)\right\},
$$

it follows that $\|K(A, \cdot)\|_{\Psi, d \omega(Q) / \omega\left(\Delta^{*}\right)} \leq T \leq C(\log 1 / \delta)^{1 / N^{\prime}} / \omega\left(\Delta^{*}\right)$. As a consequence,

$$
\begin{aligned}
V(A) & \leq C \rho(\log 1 / \delta)^{1 / N^{\prime}}\left(f_{\Delta^{*}} f_{1}^{\#}(Q)^{N} d \sigma\right)^{1 / N} \\
& \leq C \rho^{1-\gamma / N^{\prime}}\left(f_{\Delta^{*}} f_{1}^{\#}(Q)^{N} d \sigma\right)^{1 / N} \\
& \leq C\left(M_{N-\gamma N / N^{\prime}}\left(f_{1}^{\#}\right)^{N}\left(Q_{0}\right)\right)^{1 / N},
\end{aligned}
$$

where $M_{a} g(Q)=\sup _{Q \in \Delta} \mathrm{r}(\Delta)^{a} f_{\Delta}|g| d \sigma$. To deal with $\left(M f^{2}\right)^{1 / 2}\left(Q_{0}\right)$, arguing as in the proof of Theorem 5.2, we see that

$$
\left(M f^{2}\right)^{1 / 2}\left(Q_{0}\right) \leq C M f\left(Q_{0}\right)+C\left(M_{N-\gamma N / N^{\prime}}\left(f_{1}^{\#}\right)^{N}\left(Q_{0}\right)\right)^{1 / N}
$$

for all $\gamma$ with $0<\gamma \leq N^{\prime}$. Also, small modifications in the proof of Lemma 3.4 yield that

$$
M f\left(Q_{0}\right) \leq C\left(M_{N-\gamma N / N^{\prime}}\left(f_{1}^{\#}\right)^{N}\left(Q_{0}\right)\right)^{1 / N}
$$

for the same $\gamma$. Since for $f \in L^{1}$ and $0 \leq a<N$ it holds that

$$
H_{\infty}^{N-a}\left(\left\{M_{a} g>t\right\}\right) \leq C\|g\|_{1} / t,
$$

the desired weak-type inequality follows.

As mentioned in the introduction, in the $\mathbf{X}=\mathbb{R}^{N}$ case Theorem 6.1 can be improved to a strong-type inequality using the so-called strong capacitary 
estimates [NRS] (see also [Do]) and the same techniques can be used to show for Lipschitz domains $D$ that, if $f \in W^{N, 1}(\partial D)$ and $0<\gamma \leq N^{\prime}$,

$$
\int_{0}^{\infty} H_{\infty}^{\gamma N / N^{\prime}}\left(\left\{E_{\gamma, \lambda} f>t\right\}\right) t^{N-1} d t \leq C\left(\|f\|_{N}+\left\|f_{1}^{\#}\right\|_{N}\right)^{N} .
$$

However, it is not clear how to extend these methods to the case of a general space of homogeneous type.

We finally mention nontangential convergence results. In the situation of $\S 3$, setting

$$
T_{\lambda} f(x)=\sup \{|u(y, t)|: \mathrm{d}(x, y) \leq(1+\lambda) t\},
$$

we clearly have $T_{\lambda} f \leq T_{\gamma, \lambda} f$ for all $\gamma<1$ and, therefore, $\left\|T_{\lambda} f\right\|_{L^{p}\left(H_{\infty}^{m}\right)} \leq$ $C\|f\|_{p, a}$ for $Q-a p<m \leq Q$. Since the Sobolev spaces of $\S \S 4$ and 5 have all dense subsets of continuous functions, it follows that nontangential convergence holds for them except on an exceptional set of zero Hausdorff $H^{m}$-measure for all $m>Q-a p$ and $m>N-p$, respectively (i.e., of Hausdorff dimensions $Q-a p$ and $N-p$ ).

However, using the results of $\S 3$, these dimensional estimates can be improved: in fact, the exceptional set has zero $C_{a, p}$ capacity, where

$$
C_{a, p}(K)=\inf \left\{\|F\|_{p}^{p}: F \geq 0, \int_{\mathrm{d}(x, y)<1} \mathrm{~d}(x, y)^{a-Q} F(y) d y \geq \chi_{K}\right\}
$$

is the analogue in this setting of the well-known Bessel capacities (we omit the details; the $\mathbb{R}^{n}$ result is classical and see $[\mathrm{AC}]$ for the unit ball in $\mathbb{C}^{n}$ ).

\section{REFERENCES}

[AC] P. Ahern and W. Cohn, Exceptional sets for Hardy Sobolev functions, $p>1$, Indiana Univ. Math. J. 38 (1989), 417-453.

[AN] P. Ahern and A. Nagel, Strong $L^{p}$ estimates for maximal functions with respect to singular measures; with applications to exceptional sets, Duke Math. J. 53 (1986), 359-393.

[CFMS] L. Caffarelli, E. Fabes, S. Mortola and S. Salsa, Boundary behaviour of nontangential solutions of elliptic operators in divergence form, Indiana Univ. Math. J. 30 (1981), 621-640.

[CS] A. P. Calderón and R. Scott, Sobolev type inequalities for $p>0$, Studia Math. 62 (1978), 75-92.

[CF] R. Coifman and C. Fefferman, Weighted norm inequalities for maximal functions and singular integrals, Studia Math. 51 (1974), 241-250.

[CW] R. Coifman and G. Weiss, Analyse harmonique non-commutative sur certains espaces homogènes, Lecture Notes in Math., vol. 242, Springer-Verlag, 1971.

[Da1] B. E. J. Dahlberg, Estimates of harmonic measure, Arch. Rational Mech. Anal. 65 (1977), 275-288.

[Da2] _ On the absolute continuity of elliptic measures, Amer. J. Math. 108 (1986), 1119-1138.

[DVS] R. A. DeVore and R. C. Sharpley, Maximal functions measuring smoothness, Mem. Amer. Math. Soc., no. 293, 1984.

[Do] J. R. Dorronsoro, Poisson integrals of regular functions, Trans. Amer. Math. Soc. 297 (1986), 669-685.

[FJK] E. Fabes, D. Jerison and C. Kenig, Necessary and sufficient conditions for absolute continuity of elliptic-harmonic measure, Ann. of Math. (2) 119 (1984), 121-141.

[Fo1] G. B. Folland, Subelliptic estimates and function spaces on nilpotent Lie groups, Ark. Mat. 13 (1975), 161-207. 
[Fo2] _ Lipschitz classes and Poisson integrals on stratified groups, Studia Math. 66 (1979), 37-55.

[FoS1] G. B. Folland and E. M. Stein, Estimates for the $\overline{\partial_{b}}$ complex and analysis on the Heisenberg group, Comm. Pure Appl. Math. 27 (1974), 429-522.

[FoS2] _ _ Hardy spaces on homogeneous groups, Princeton Univ. Press, Princeton, N.J., 1982.

[Go] M. S. Gowda, Doctoral dissertation, Univ. of Wisconsin, Madison, 1982.

[Hd] L. I. Hedberg, On certain convolution inequalities, Proc. Amer. Math. Soc. 36 (1972), 505510 .

[He] S. Helgason, Differential geometry, Lie groups and symmetric spaces, Academic Press, New York, 1978.

[JK] D. Jerison and C. Kenig, Boundary behavior of harmonic functions in nontangentially accessible domains, Adv. in Math. 46 (1982), 80-147.

[KV] A. Korányi and S. Vági, Singular integrals in homogeneous spaces and some problems of classical analysis, Ann. Scuola Norm. Sup. Pisa 25 (1971), 575-648.

[NRS] A. Nagel, W. Rudin and J. Shapiro, Tangential behavior of functions in Dirichlet-type spaces, Ann. of Math. (2) 116 (1982), 331-360.

[NS] A. Nagel and E. M. Stein, On certain maximal functions and approach regions, Adv. in Math. 54 (1984), 83-106.

[St] E. M. Stein, Singular integrals and differentiability properties of functions, Princeton Univ. Press, Princeton, N.J., 1970.

[Su] J. Sueiro, Tangential boundary limits and exceptional sets for holomorphic functions in Dirichlet-type spaces, Math. Ann. 286 (1990), 661-678.

[Z] A. Zygmund, Trigonometric series, Cambridge Univ. Press, London and New York, 1959.

(P. Cifuentes and J. R. Dorronsoro) Facultad de Ciencias, Departamento de Matemáticas, Universidad Autónoma de MadRID 28049 Madrid, SPaIN

(Juan Sueiro) Departamento de Matemáticas, Universidad de Barcelona, 08071, BarceLONA, SPAIN 